\title{
Genetic variability of host-parasite relationship traits: utilization of isofemale lines in a Drosophila simulans parasitic wasp
}

\author{
Y. Carton ${ }^{1 *}$, P. Capy ${ }^{1}$ and A.J. Nappi ${ }^{2}$ \\ ${ }^{1}$ Centre National de la Recherche Scientifique, Laboratoire de Biologie et Génétique \\ Evolutives, 91198 Gif-sur-Yvette Cédex, France; \\ ${ }^{2}$ Department of Biology, Loyola University of Chicago, Chicago, IL 60626, USA
}

(received 3 November 1988; accepted 21 August 1989)

Summary - We investigated genetic variability of traits involved in the successful parasitization of larvae of Drosophila melanogaster and $D$. simulans by the hymenopteran parasite Leptopilina boulardi. Characters studied were: the rate of infestation, overall developmental success, ability to escape host encapsulation, developmental success after eclosion, and physiological incompatibility between the 2 partners. These investigations were performed over 3 generations (G1, G2 and G4) using 14 isofemale lines of $L$. boulardi collected in Tunisia. The host was $D$. simulans. For the first 4 traits, the mean values were relatively constant from 1 generation to another. Comparisons of variability within and between isofemale lines of the same generation, and correlations between generations, indicate a genetic component for 2 traits: overall developmental success and ability to evade encapsulation.

Drosophila parasitoids - host infestation - developmental success - encapsulation escape - genetic variability

Résumé - Variabilité génétique dans les relations hôte-parasitoïde: utilisation des lignées isofemelles chez un hyménoptère parasite de drosophile. La variabilité génétique de caractères impliqués dans le succès d'infestation de larves de Drosophila melanogaster et de D. simulans par Leptopilina boulardi, un hyménoptère parasite, a été entreprise. Les caractères étudiés étaient: le taux d'infestation, le succès de développement global, l'aptitude à éviter l'encapsulation par l'hôte, le succès de développement après éclosion et l'incompatibilité physiologique entre les 2 partenaires. Cette analyse a été réalisée sur 3 générations (G1, G2 et G4) à partir de 14 lignées isofemelles de L. boulardi originaires de Tunisie. L'hôte utilisé ici, était $\mathrm{D}$. simulans. Les moyennes des 4 premiers caractères restent stables au cours des générations. La comparaison des variabilités intra- et interlignées au sein d'une même génération et les corrélations entre les générations révèlent l'existence d'une importante composante génétique pour deux caractères: le succès de développement global et l'aptitude à éviter l'encapsulation.

parasites de drosophiles - taux d'infestation - succès de développement - évitement de la réaction de l'hôte - variabilité génétique

* Correspondence and reprints. 


\section{INTRODUCTION}

Very few investigations concern the genetic bases of the adaptations acquired by hosts or parasites that contribute to the successful development of these competitively interacting partners during their coevolution. The haploid-diploid reproduction of Hymenoptera precludes application of the usual procedures for quantitative genetic analysis. One of the methods of quantitative genetic analysis available for such studies involves the use of isofemale lines, i.e. the progeny derived from a single female inseminated in nature (Parsons and Hosgood, 1967; David, 1979; Wallis et al., 1985). The isofemale line method has been successfully employed in analysis of the genetic variability of various traits in Drosophila species (see Parsons, 1980 for a review). Previous studies by Bouletreau and Fouillet (1982) and Bouletreau (1986) showed a wide variation of host suitability among isofemale lines of Drosophila in a single generation. Recently, using this technique, Carton and Boulétreau (1985) demonstrated a genetic basis for the ability of host larvae of Drosophila melanogaster to encapsulate and destroy eggs of parasitic wasps.

In order to ascertain the relative importance of genetic components involved in the successful development of a parasite (or in the acquisition of an effective immune response by the host), comparisons of the variability between and within isofemale lines can be used: a significantly higher variability among lines than within lines probably indicates genetic differences among lines. In addition, correlations between successive generations may also provide some estimates of the heritability of quantitative traits. In the present work, both approaches were used to estimate the genetic variability of several traits involved in the successful development of the Cynipid wasp Leptopilina boulardi (Barbotin et al., 1979), new comb. (Nordlander, 1980 ), a specific parasite of the sibling species $D$. melanogaster and $D$. simulans.

\section{MATERIALS AND METHODS}

\section{General procedures}

The 14 isofemales lines of $L$. boulardi used in this study were caught in the oasis of Nasrallah (near Kairouan, Tunisia) in 1985 and have been reared on a strain of $D$. melanogaster collected from the same location. At this site, D. simulans is more common and more abundant than $D$. melanogaster throughout the year and probably represents the main host for $L$. boulardi (Carton et al., 1986, 1987). Therefore, we used $D$. simulans as a host in this study.

Genetic variability of different traits was calculated in first (G1), second (G2) and fourth (G4) generations of parasites reared under laboratory conditions. In addition, to minimize host variation, all the $D$. simulans used in the study were recently derived from a single female also originating from Nasrallah.

Five batches of 100 Drosophila eggs (0-6 h old) were put in vials containing a killed yeast medium (David and Clavel, 1965) and maintained at $25^{\circ} \mathrm{C}$. Twentyfour hours later, the larvae of 4 batches were exposed to a single $L$. boulardi female for a period of $24 \mathrm{~h}$. The fifth batch (unexposed larvae) served as control. Four inseminated wasp females, 5-8 days old, were tested, from each of the 14 isofemale lines. Only 'experienced' wasps were used; before each experiment the parasites were 
allowed to parasitize $D$. simulans larvae for $4 \mathrm{~h}$, and thus acquired prior oviposition experience with this host (van Lenteren, 1981). Following development at $25^{\circ} \mathrm{C}$, the adult flies and wasps were counted and examined. Hosts that had produced an immune response were identified by the presence of melanotic capsules within their abdomen (Carton and Kitano, 1981; Carton et al., 1986).

\section{Calculation of biological parameters}

Four different parameters were recorded for each test (Fig. 1):

- the number of adult flies without melanotic capsule $(A)$;

- the number of adult flies containing a dead, melanized and encapsulated parasite in the abdomen $(B)$;

- the number of adult wasps $(C)$;

- the number of dead Drosophila larvae or pupae $(D)$. This parameter, $D$, is equal to $100-(A+B+C)$.

Previous studies (Carton, 1984) have shown that, except for the process of melanotic encapsulation, there is no abortive development of the parasite egg in the case of sympatric infestations. Also, mortality of a host containing an encapsulated egg is not enhanced by the occurrence of this foreign body. Larvae unexposed to the wasps served as controls. By subtracting the number of dead fly larvae and pupae recorded in the controls $(E)$ from the number of dead fly larvae and pupae recorded in the tests $(D)$, a measure of host mortality due to the parasite was obtained.

For each test, two other parameters were calculated as follows:

- the number $(Y)$ of potential hosts

$Y=A+B+C+(D-E)$

- the number $(X)$ of infested hosts

$$
X=B+C+(D-E)
$$

The following quantitative parameters, for which the genetic variability was calculated, provide an estimate of the effectiveness of the parasite. These parameters are not totally independent but each provides some different information:

- Infestation ability: Inf. Ab. $(\%)=(X / Y) 100$

- Overall developmental success: Ov. Dev. Suc. $(\%)=(C / X) 100$

- Ability to evade encapsulation: Ab. Ev. Enc. $(\%)=(1-(B / X)) 100$

- Developmental success after eclosion: Dev. Suc. Ec. $(\%)=(C /(C+(D-E))) 100$

- Degree of incompatibility between host and parasite: Deg. Inc. $(\%)=$ $((D-E) / X) 100$

\section{Statistical methods}

For the 3 generations, means and variances of each isofemale line and mean squares within and among lines were calculated for the 5 previous parameters. Moreover, to make the means and variances of the different values independent, we performed all statistical tests on arcsin-transformed values.

At each generation, analysis of variance was used to estimate the within $\left(V_{w}\right)$ and the between $\left(V_{b}\right)$ components of the total variance $\left(V_{t}=V_{w}+V_{b}\right)$. From these 


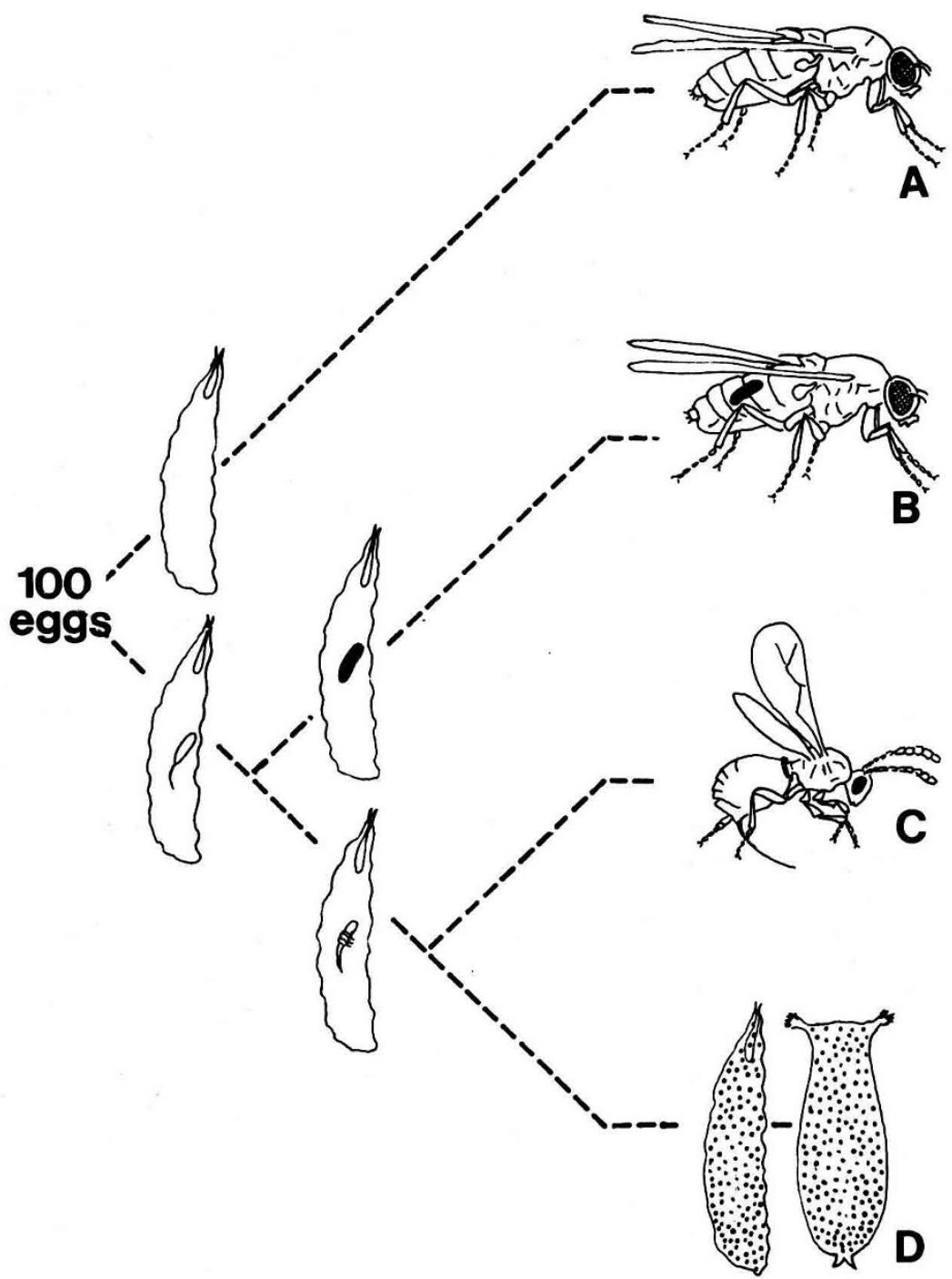

Fig. 1. Representation of the issue of host larvae subject to infestation by the parasitic wasp $L$. boulardi.

components it is then possible to estimate the intraclass correlation $\left(t=V_{b} / V_{t}\right)$. This latter parameter estimates the average similarity of individuals belonging to the same group (Falconer, 1981), i.e. in the present work, individuals belonging to isofemale lines. Hoffman and Parsons (1988) call this parameter (when using isofemale lines) the 'isofemale heritability'. As with the 'populational heritability' defined by Slatkin (1981), this heritability is intermediate between a heritability in a narrow sense $\left(h^{2}\right)$ and a heritability in a broad sense $\left(H^{2}\right)$. 
The confidence interval of this heritability depends on the confidence interval of the intraclass correlation. In the present paper, the following expression will be used:

$$
\operatorname{var}(t)=\frac{2(1-t)^{2}(1+(n-1) t)}{n(n-1)(N-1)}
$$

where $n$ is the number of individuals measured per line and $N$ the number of lines (Bulmer, 1985; Donner and Wells, 1986).

The correlation of the parameters studied between successive generations provides another approach to the heritability of a trait. In our case, we only have the means of each isofemale line. Therefore, it is not possible to estimate accurately the degree of relatedness between 2 individuals belonging to the same line and/or individuals of different generations. However, as stressed by Capy (1987), if the effective population size of each line is not too small, the correlation of the parameters studied between successive generations can be used as an estimate of the upper limit of the heritability.

\section{RESULTS}

\section{Means and variances of the parameters}

Table I gives the means and coefficients of variation of the 5 parameters measured. For most of the traits, the mean values are stable between successive generations. As shown by the data on extreme values, the distributions of some parameters are asymmetrical.

Results of the 2-way analysis of variance are given in Table II. The total variance has been partitioned into mean squares among lines, among generations, lines $\times$ generations interaction and a residual component. For all the 5 characters, the isofemale line effect is significant, suggesting at least partial genetic causes of the differences among lines. It also appears that for 3 traits (Dev. Suc. Ecl., Deg. Inc. and Ov. Dev. Suc.) there is a significant generation effect. These last results may be explained by uncontrolled environmental variation among generations.

\section{Genetic variability}

Estimates of intraclass correlation are given in Table III. Among the 5 parameters which show an isofemale effect (see below), only 2 of them present high values of intraclass correlation regardless of the generation. The overall developmental success shows stable values over generations. For the other traits, some important variation may exist among generations. Especially for the Ability to Evade Encapsulation, high values of this parameter are observed in G2 and G4 but not in G1. As stated in 'Materials and Methods', the intraclass coefficient $(t)$ provides estimates of the heritability. This estimation, given by $h^{2}=2 t$ (Falconer, 1981), provides values of the upper limits of heritability. In this case, the genetic variability will include not only additive effects but possibly some dominance effects well. Moreover, it is possible that the among line variance may overestimate the genetic variance, as it may include uncontrolled factors such common environmental effects ('vial effects'). 
Table I. Means (original data) and standard errors (expressed in \%) of the 5 parameters studied over 3 generations (G1, G2 and G4). ${ }^{a}$

\begin{tabular}{lccc}
\hline Character & $G 1$ & $G 2$ & $G 4$ \\
\hline Inf. Ab. & $71.5 \pm 2.4$ & $65.2 \pm 2.1$ & $70.4 \pm 2.2$ \\
& $(60.4-94.9)$ & $(44.9-81.8)$ & $(50.5-82.5)$ \\
Ov. Dev. Suc. & 25.3 & 24.5 & 29.5 \\
& $72.0 \pm 1.6$ & $72.2 \pm 2.7$ & $63.9 \pm 2.6$ \\
& $(65.7-82.4)$ & $(35.2-87.6)$ & $(26.6-79.9)$ \\
Ab. Ev. Enc. & 16.3 & 27.9 & 31.2 \\
& $91.2 \pm 1.2$ & $89.1 \pm 2.1$ & $93.3 \pm 1.3$ \\
& $(84.3-97.3)$ & $(84.4-98.5)$ & $(90.4-99.6)$ \\
Dev. Suc. Ecl. & 9.7 & 17.6 & 10.0 \\
& $79.4 \pm 1.6$ & $78.3 \pm 2.5$ & $67.6 \pm 2.6$ \\
Deg. Inc. & $(69.7-93.8)$ & $(47.5-91.2)$ & $(27.4-82.1)$ \\
& 15.3 & 24.0 & 29.4 \\
& $19.3 \pm 1.4$ & $16.8 \pm 1.6$ & $29.4 \pm 2.6$ \\
& $(6.2-33.6)$ & $(8.2-30.9)$ & $(17.4-70.5)$ \\
\hline
\end{tabular}

a Extreme values of each distribution are given in brackets. Italic values represent the coefficient of variation.

Table II. Test of the isofemale line, generation and isofemale line $\times$ generation effects by a 2-way analysis of variance with interaction. ${ }^{a}$

\begin{tabular}{lccc}
\hline Character & $\begin{array}{c}\text { Isofemale line } \\
(13,126)\end{array}$ & $\begin{array}{c}\text { Generation } \\
(2,126)\end{array}$ & $\begin{array}{c}\text { Interaction } \\
(26,126)\end{array}$ \\
\hline Inf. Ab. & $1.95^{*}$ & $2.53 \mathrm{NS}$ & $1.33 \mathrm{NS}$ \\
Ov. Dev. Suc. & $4.61^{* * *}$ & $4.14^{*}$ & $1.47 \mathrm{NS}$ \\
Ab. Ev. Enc. & $5.45^{* * *}$ & $2.14 \mathrm{NS}$ & $1.03^{*}$ \\
Dev. Suc. Ecl. & $2.88^{* * *}$ & $6.91^{* * *}$ & $1.64^{*}$ \\
Deg. Inc. & $2.82^{* * *}$ & $12.00^{* *}$ & $1.74^{*}$ \\
\hline
\end{tabular}

${ }^{a}$ Over the 3 generations, 14 isofemale lines and 4 repetitions per line were performed. Degrees of freedom are given in brackets. The variance analysis was performed on arc-sin transformed data.

Level of significance: ${ }^{*} 0.05 ;{ }^{* *} 0.01 ;{ }^{* * *} 0.001$.

Another technique to determine whether a genetic component is partly responsible for the phenotypic variability of a given trait is to consider the correlation between generations. These correlations measure the degree of ressemblance between parents and their offspring or their grand-offsprings. Results are presented in Table IV. Again, the 2 traits which previously showed high values of intraclass correlation (Ov. Dev. Suc. and Ab. Ev. Enc.) also exhibit positive and significant correlations by this statistical test. Moreover, the observed values are around or above 0.5 , suggesting a high heritability of these 2 traits. For the other traits, average correlations are much lower and vary sporadically. For example, for Inf. Ab., the values range between -0.01 and 0.5 . These results are complementary to those given in Table III. 
Table III. Estimates (calculated from arc-sin transformed data) of intraclass correlations $(t)$ were calculated by the ratio $V_{b} / V_{b}+V_{w}$ ) where $V_{b}$ and $V_{w}$ are the between and the within-line components of the total variance.

\begin{tabular}{lcccccc}
\hline Traits & \multicolumn{2}{c}{ G1 } & \multicolumn{2}{c}{ G2 } & \multicolumn{2}{c}{ G4 } \\
& \multicolumn{1}{c}{$t$} & \pm S.E. $(t)$ & \multicolumn{1}{c}{ \pm . } & & \multicolumn{1}{c}{$t(t)$} & $\pm . E .(t)$ \\
\hline Inf. Ab. & 0.11 & 0.13 & 0.29 & 0.15 & -0.03 & 0.12 \\
Ov. Dev. Suc. & 0.24 & 0.15 & 0.28 & 0.15 & 0.28 & 0.15 \\
Ab. Ev. Enc. & -0.01 & 0.11 & 0.31 & 0.15 & 0.50 & 0.14 \\
Dev. Suc. Ecl. & 0.15 & 0.14 & 0.17 & 0.14 & 0.27 & 0.15 \\
Deg. Inc. & 0.31 & 0.15 & 0.09 & 0.13 & 0.23 & 0.15 \\
\hline
\end{tabular}

The standard errors of $t$ were calculated from the expression given in the text where $n=4$ and $N=14$.

Table IV. Correlations between the different generations calculated from the mean values of the fourteen isofemale lines.

\begin{tabular}{llcc}
\hline Character & G1-G2 & G2-G4 & G1-G4 \\
\hline Inf. Ab. & $0.50^{+}$ & -0.01 & 0.27 \\
Ov. Dev. Suc. & $0.69^{* *}$ & $0.52{ }^{+}$ & $0.49^{+}$ \\
Ab. Ev. Enc. & $0.51^{+}$ & $0.81^{* * *}$ & $0.53^{*}$ \\
Dev. Suc. Ecl. & $0.31^{+}$ & 0.10 & 0.26 \\
Deg. Inc. & 0.30 & -0.01 & 0.27 \\
\hline
\end{tabular}

Level of significance: ${ }^{+} 0.10 ;^{*} 0.05 ;{ }^{* *} 0.01 ;{ }^{* * *} 0.001$.

\section{DISCUSSION AND CONCLUSION}

The infestation ability (Inf. Ab.) measures the ethological efficiency of the female wasp to parasitize host larvae. Carton (1984) demonstrated previously that there is no abortive development of a parasite egg except by the process of encapsulation, since the level of infestation so estimated was not different from the level evaluated by dissection in the case of sympatric infestations.

The overall developmental success (Ov. Dev. Suc.) of the parasite includes the physiological traits contributing to the successful development of the parasite (including evading encapsulation). The ability to evade encapsulation ( $\mathrm{Ab}$. Ev. Enc.) is useful for entomophagous species since all insect larvae (Gotz, 1986) and especially Drosophila larvae (Nappi and Carton, 1986) are able to encapsulate nonself material. Despite the apparent efficiency of Drosophila host defense mechanisms, parasitic wasps are able in some conditions to evade this process (Rizki and Rizki, 1984).

The developmental success after eclosion (Dev. Suc. Ec.) measures the suitability of the host for the parasite. The encapsulation process, when present, occurs on parasite egg instar; these eggs attacked will die later. Mortality of the host larva containing an encapsulated egg in its cavity is not enhanced by the occurrence of this foreign body. Preliminary experiments, performed with 320 larvae submitted to infection, showed that the rate of immune reaction, estimated from flies with 
capsules (11.5\%), was not lower than the actual rate evaluated by the dissection of Drosophila larvae $48 \mathrm{~h}$ after infestation (10.9\%).

The degree of incompatibility between the host and the parasite (Deg. Inc.) measures the proportion of host larvac which die because of live parasites. An acute incompatibility was already been shown between host and parasite strains of different geographic origins (Carton, 1984).

Studies of the genetic variability among and within isofemale lines of successive generations of $L$. boulardi indicate that 2 traits are heritable: the overall developmental success and the ability of the parasite to evade encapsulation. For the other three traits, our estimates of heritability are not significantly different from zero. Indeed, for these latter traits, it is likely that strong uncontrolled environmental effects exist. For the moment, we cannot conclude that there is no heritability for these traits, but only that it may be masked by environmental variability.

Based on our results, we suggest that an improvement of the overall developmental success of parasitism could be obtained by artificial or natural selection. In fact, the target of selection concerns the ability to evade encapsulation which presents a high potential heritability (Tables III and IV). Resistant genes may be involved in the production or the regulation of a factor playing a role in the protective process. This factor could correspond to the inhibitor factor I (Walker, 1959) or to the recently discovered lamellolysin (Rizki and Rizki, 1984), a factor responsible for host lamellocyte destruction.

Previous investigations have been performed on the genetic basis of parasitization traits. Investigating the ethological aspects of the primary sequences of infestation, Chabora (1967) compared 2 geographic lines of the parasite Nasonia vitripennis, but found no genetic differences in the level of infestation. On the other hand, he detected a genetic difference in the proportion of adjacent hosts attacked, but this difference decreased from the first to the fourth generation, perhaps because the selection pressure was removed. Samson-Boshuizen et al. (1974) discovered some differences in parasitization behaviour between 2 geographical strains of Leptopilina heterotoma. A Swiss strain was found to be less efficient in infection capacity than a USA strain. Our results do not demonstrate the existence of a genetic basis for infestation ability.

Veerkamp (1982) observed in L. heterotoma that differential mortality of parasitized hosts was caused by differences in genetical background among the wasp strains. A similar process (Carton, 1984) was observed in a comparison of strains (Guadeloupe and Brazil) of $L$. boulardi. The present study, however, failed to demonstrate clearly the existence of important genetic variability for this trait (Deg. Inc.) in a natural population. However, the ability to evade encapsulation appears to have a strong genetic component. Convincing results have been obtained over three successive generations. Walker (1959) reported differences among geographic strains of $L$. heterotoma for their sensitivity to the host encapsulation process.

The overall developmental success depends on the ability to evade encapsulation during the embryonic stage and from the developmental success after eclosion during the larval period. Indeed, correlations between these traits (Ov. Dev. Suc. - Ab. Ev. Enc., $r=0.51$; Ov. Dev. Suc. - Dev. Suc. Ec., $r=0.88$ with 40 d.f.) are highly significant. The present results provide evidence that the observed genetic component of overall developmental success is of similar magnitude to that of 
the ability to evade encapsulation. However, data on developmental success after eclosion do not show a significant genetic variability in our experiments. It is also important to note that a very high within-strain variance may hide the among-strain genetic component of a quantitative trait.

\section{ACKNOWLEDGEMENTS}

We thank Dr J. David who made helpful comments on the manuscript. We are also very grateful to Mrs F. Frey for technical assistance. This research was supported by a research grant from the CNRS (ATP Biologie des populations 900227).

\section{REFERENCES}

Barbotin F., Carton Y. \& Kelner-Pillault S. (1979) Morphologie et biologie de Cothonaspis (Cothonaspis) boulardi n.sp., parasites des Drosophiles. Bull. Soc. Ent. Fr. 84, 19-26

Boulétreau M. (1986) The genetic and coevolutionary interactions between parasitoids and their hosts. In: Insect Parasitoids (J. Waage and D. Greathead, eds), Academic Press, London, pp. 169-200

Boulétreau M. \& Fouillet M. (1982) Variabilité génétique intrapopulation de l'adéquation de Drosophila melanogaster à un de ses parasites hyménoptères. C.R. Acad. Sci. 295, 775-778

Bulmer M.G. (1985) The Mathematical Theory of Quantitative Genetics, Clarendon Press, Oxford

Capy P. (1987) Variabilité génétique des populations naturelles de Drosophila melanogaster et de Drosophila simulans. Thèse, University of Paris

Carton Y. (1984) Analyse expérimentale de trois niveaux d'interactions entre Drosophila melanogaster et le parasite Leptopilina boulardi (sympatrie, allopatrie, xénopatrie). Génét. Sél. Evol. 4, 417-430

Carton Y.\& Boulétreau M. (1985) Encapsulation ability of Drosophila melanogaster: a genetic analysis. Dev. Comp. Immunol. 9, 1-10

Carton Y., Boulétreau M., van Alphen J.J.M. \& van Lenteren J.C. (1986) The Drosophila parasitic wasps. In: The Genetics and Biology of Drosophila (M. Ashburner, H.L. Carson \& J.N. Thompson, eds), vol. 3, Academic Press, London, pp. 347-394

Carton Y., Chibani F., Haouas S. \& Marrakchi M. (1987) Egg-laying strategy under natural conditions of Leptopilina boulardi, a hymenopteran parasite of Drosophila spp. Entomol. Exp. Appl. 43, 193-201

Carton Y. \& Kitano H. (1981) Evolutionary relationships to parasitism by seven species of the Drosophila melanogaster subgroup. Biol. J. Linn. Soc. 16, 350-362

Chabora P.C. (1967) Hereditary behavior variation in oviposition patterns in the parasite Nasonia vitripennis (Hymenoptera: Pteromalidae). Canadian Entomologist 99, 763-765 
David J. (1979) Utilization of morphological traits for the analysis of genetic variability in wild populations. Aquilo. Ser. Zool. 20, 49-61

David J. \& Clavel M.F. (1965) Intéraction entre le génotype et le milieu d'élevage. Conséquences sur les caractéristiques du développement de la drosophile. Bull. Biol. Fr. Belg. 99, 369-378

Donner A. \& Wells G. (1986) A comparison of confidence interval methods for the intraclass correlation coefficient. Biometrics 42, 401-412

Falconer D.S. (1981) Introduction to Quantitative Genetics, Longman, London Gotz P. (1986) Encapsulation in Arthropods. In: Immunity in Invertebrates (Brehelin M., ed.), Springer-Verlag, Berlin, pp. 155-170

Hoffmann A.A. \& Parsons P.A. (1988) The analysis of quantitative variation in natural populations with isofemale strains. Genet. Sél. Evol. 20, 87-98

Lenteren J.C. van (1981) Host discrimination by parasitoïds. In: Semiochemicals: Their Role in Pest Control (D.A. Nordlund, R.L. Jones and W.J. Lewis, ed.), Wiley, NY, pp. 153-179

Nappi A. \& Carton Y. (1986) Cellular immune response and their genetic aspects in Drosophila. In: Immunity in Invertebrates (Brehelin M., ed.), Springer-Verlag, Berlin, pp. 171-187

Nordlander G. (1980) Revision of the genus Leptopilina Forster, 1869, with notes on the status of some other genera (Hymenoptera, Cynipoidea: Eucoilidae). Entomol. Scand. 11, 428-453

Parsons P.A. (1980) Isofemale strains and evolutionary strategies in natural populations. Evol. Biol. 13, 175-217

Parsons P.A. \& Hosgood S.M.W. (1967) Genetic heterogeneity among the founders of laboratory populations of Drosophila. Genetica 38, 328-339

Rizki T.M. \& Rizki R.M. (1984) Selective destruction of a host blood cell type a parasitoid wasp. Proc. Natl. Acad. Sci. USA 81, 6154-6158

Samson-Boshuizen M.H., van Lenteren J.C. \& Bakker K. (1974) Success of parasitization of Pseudeucoila bochei Weld (Hym. Cynipidae): a matter of experience. Neth. J. Zool. 24, 67-85

Slatkin M. (1981) Fixation probabilities and fixation times in a subdivided population. Evolution 35, 477-488

Veerkamp F.A. (1982) Genetic variation in the pattern of initial oviposition behaviour of Leptopilina heterotoma Thompson (= Pseudeucoila bochei Weld), a parasite of Drosophila melanogaster. Neth. J. Zool. 32, 88-107

Walker I. (1959) Die Abwehrreaktion des Wirtes Drosophila melanogaster gegen die Zoophage Cynipidae Pseudeucoila bochei Wald. Rev. Suisse Zool. 66, 569-632

Wallis G.P., Aitken T.H.G., Beaty B.J., Lorenz L., Amato G.D. \& Tabachnick W.J. (1985) Selection for susceptibility and refractoriness of Aedes aegypti to oral infection with yellow fever virus. Am. J. Trop. Med. Hyg. 34, 1225-1231 